University of Texas at El Paso

ScholarWorks@UTEP

3-1998

\title{
How to Divide a Territory? A New Simple Differential Formalism for Optimization of Set Functions
}

Hung T. Nguyen

Vladik Kreinovich

The University of Texas at El Paso, vladik@utep.edu

Follow this and additional works at: https://scholarworks.utep.edu/cs_techrep

Part of the Computer Engineering Commons

Comments:

Technical Report: UTEP-CS-97-25a

Published in International Journal of Intelligent Systems, 1999, Vol. 14, No. 3, pp. 223--251.

\section{Recommended Citation}

Nguyen, Hung T. and Kreinovich, Vladik, "How to Divide a Territory? A New Simple Differential Formalism for Optimization of Set Functions" (1998). Departmental Technical Reports (CS). 546.

https://scholarworks.utep.edu/cs_techrep/546

This Article is brought to you for free and open access by the Computer Science at ScholarWorks@UTEP. It has been accepted for inclusion in Departmental Technical Reports (CS) by an authorized administrator of ScholarWorks@UTEP.For more information, please contact Iweber@utep.edu. 


\title{
How to Divide a Territory? A New Simple Differential Formalism for Optimization of Set Functions
}

\author{
Hung T. Nguyen ${ }^{1}$ and Vladik Kreinovich ${ }^{2}$ \\ ${ }^{1}$ Department of Mathematical Sciences \\ New Mexico State University \\ Las Cruces, NM 88003 \\ email hunguyen@nmsu.edu \\ ${ }^{2}$ Department of Computer Science \\ University of Texas at El Paso \\ El Paso, TX 79968 \\ email vladik@cs.utep.edu
}

\begin{abstract}
In many practical problems, we must optimize a set function, i.e., find a set $A$ for which $f(A) \rightarrow \max$, where $f$ is a function defined on the class of sets. Such problems appear in design, in image processing, in game theory, etc.

Most optimization problems can be solved (or at least simplified) by using the fact that small deviations from an optimal solution can only decrease the value of the objective function; as a result, some derivative must be equal to 0 . This approach has been successfully used, e.g., for set functions in which the desired set $A$ is a shape, i.e., a smooth (or piece-wise smooth) surface. In some real-life problems, in particular, in the territorial division problem, the existing methods are not directly applicable. For such problems, we design a new simple differential formalism for optimizing set functions.
\end{abstract}




\section{Introduction: Optimization of Set Functions is a Practically Important but Difficult Prob- lem}

Optimization is important. In most application problems, we have a class $\mathcal{A}$ of possible alternatives, and we must find the best of these alternatives, i.e., the alternative $a \in \mathcal{A}$ for which the given objective function $f: \mathcal{A} \rightarrow R$ takes the largest possible value:

$$
f(a) \rightarrow \max _{a \in \mathcal{A}} .
$$

Example: optimization is important in statistics. An important particular case of this problem is when the objective function $f(a)$ describes the posterior probability (likelihood) of an alternative $a$; the corresponding optimization problem is called the maximum likelihood method (MLM) of choosing an alternative.

Simplest case: finitely many alternatives. The larger the class of alternatives $\mathcal{A}$, the more complicated the corresponding optimization problem.

The simplest case is when we have finitely many alternatives; this happens, e.g., when we compare several proposed designs for a new car, or several proposed plans of economic development, etc. In this case, we can simply try all of them, and choose the one for which the objective function takes the largest possible value.

The very fact that this case if the simplest does not mean that it is easy: In many real-life problems, we have several features to choose; even if we have only two options for each of $n$ feature, we have $2^{n}$ possible choices; for large $n$, this number grows so fast that exhaustive search becomes practically impossible. Moreover, for some finite optimization problems (called NP-hard), not only exhaustive search is computationally impossible, but any other algorithm requires, for some instances at least, the unrealistic exponential time (see, e.g., $[22,57])$.

Next simplest case: optimization of functions of one or several variables. The simplest case is when alternatives from the class $\mathcal{A}$ can be uniquely characterized by a single real-valued parameter $x$, or by finitely many realvalued parameters $x_{1}, \ldots, x_{n}$. Since the alternative $a$ is uniquely determined by the values of these parameters, the value of the objective function $f(a)$ (that describes the relative quality of different alternatives) is also uniquely determined by these values: $f(a)=F\left(x_{1}, \ldots, x_{n}\right)$ for some known (and everywhere defined) function $F\left(x_{1}, \ldots, x_{n}\right)$ of $n$ real variables. In this case, finding the best alternative means finding the values $x_{1}, \ldots, x_{n}$ for which $F\left(x_{1}, \ldots, x_{n}\right) \rightarrow \max$.

In spite of its perceived simplicity, in some cases, this problem is extremely computationally complicated (see, e.g., [35, 58, 67]), However, usually, this optimization problem is simplified by the fact that the function $F\left(x_{1}, \ldots, x_{n}\right)$ if 
smooth (differentiable). This means, in particular, that its maximum is located at a point where all $n$ partial derivatives $\partial F / \partial x_{i}$ are equal to 0 .

- In some cases, the resulting $n$ equations $\partial F / \partial x_{i}=0$ are sufficient to find $x_{i}$.

- In more complicated cases, these equations are not sufficient to immediately find the desired values $x_{i}$, but we can use the partial derivatives to design an iterative method that converges to the desired values $x_{i}$ really fast. For example, we can use a gradient method in which the next iteration $x_{i}^{\prime}$ is obtained from the previous iteration $x_{i}$ by a formula $x_{i}^{\prime}=x_{i}-\lambda \cdot \partial F / \partial x_{i}$ (or a modification of this method).

In all these cases, differentiability helps.

Optimization of functionals. An even more complex case if when the alternatives cannot be characterized by finitely many parameters, but require a function (or finitely many functions) to describe. A typical example is theoretical physics, where most fundamental theories are formulated in terms of the so-called variational principles, i.e., principles of the type $S(a) \rightarrow \max$, where $a$ denotes a function (physical field $\varphi(x)$ ) or several fields, and $S(a)$ is a functional called action.

For example, General Relativity Theory is formulated in this form with $a$ equal to the metric field $g_{i j}(x)$ and $S=\int R \cdot \sqrt{-g} d^{4} x$. Most modern physical theories are formulated in terms of the so-called Lagrangian, i.e., a function $L$ for which $S=\int L d^{4} x$.

To find solutions to the corresponding variational problem, we can use the formalism of variational (functional) derivatives and equate the corresponding functional derivatives with 0 . As a result, for all classical theories, we get the corresponding partial differential equations (see, e.g., [18, 37]).

If we cannot directly solve these equations, we can use the corresponding version of an iterative gradient method; this methods is often indeed very successful; e.g., in [62], we successfully used it in radioastronomy.

Optimizing set functions is necessary. In some practical problems, alternatives are described by sets. In this case, the objective function $f(a)$ is defined on a class of sets. Such functions are called set functions. Thus, in these problems, we must optimize set functions.

There are several important classes of problems in which alternatives are sets:

- In design, we must find the best shape of the designed object: e.g., the aerodynamically best shape of a plane, of a car, or of a Space Shuttle.

- In image processing, we must extract, from the observed noisy data, the best image to fit the observations. Often, we do not have enough information to reconstruct the grey levels of different image pixels; in this case, we 
are interested in reconstructing a black-and-white (binary) image. A binary image is, in essence, a set: it can be uniquely described if we describe the set of all black pixels.

- Another important class of practical optimization problems in which sets naturally appear is problems of territorial division. These problems range from big political problems, in which a disputed territory has to be divided between contesting countries to everyday economic problems, in which, e.g., a large tract of agricultural land, with varying properties at different points, has to be divided into areas of different usage: some pieces allocated for different crops, some for cattle pastures, some should not be used, etc. For each of the usages, we want to describe the corresponding set of all points reserved for this particular usage.

In territorial division problems, there are usually several sides with conflicting interests, and therefore, we must use the theory of such conflict situations, usually known as game theory $[40,51]$, to select an appropriate objective function.

Similarly to all other cases, an important particular case of set function optimization is when the objective function $f(a)$ describes the posterior probability (likelihood) of an alternative $a$, i.e., when we use the maximum likelihood method to choose a set based on a given probability measure on the class of all sets (i.e., on a random set); for examples and detailed exposition, see, e.g., [24] and references therein.

Optimizing set functions is difficult. Anyone who has tried to optimize a set function knows that it is often a very computationally difficult problem. There are two reasons for this difficulty:

- First, there is a general reason that is equally applicable both to sets and to functions: The more alternatives we have, the more complicated the corresponding optimization problem. Therefore, problems in which alternatives are functions or sets are, in general, much more difficult to solve than problems in which alternatives can be characterized by finitely many parameters.

- Second, there is a specific reason which makes sets more difficult alternatives than functions: Sets can be described by their characteristics functions, and so optimizing over sets is the same as optimizing over functions with an additional constraint that a function cannot take arbitrary real values, only values from the set $\{0,1\}$. At first glance, this constraint restricts the set of possible alternatives and thus, it should make the optimization problem easier, but in reality, it is much easier to optimize over continuously changing variables than over variables with a discrete range like $\{0,1\}$. 
This relative complexity can be traced to the simplest case when we only consider subsets of a finite universal set. In this case, a characteristic function $x$ is simply a finite sequence of binary numbers $x_{1}, \ldots, x_{n} \in$ $\{0,1\}$, where $n$ is the total number of elements in the universal set. Even for linear objective functions $f(x)=\sum s_{i} \cdot x_{i}$, checking whether 0 is a possible value of this function is an NP-hard (computationally intractable) problem [22], while for continuous variables (e.g., variables running over intervals $\left.\left[\underline{x}_{i}, \bar{x}_{i}\right]\right)$, it is computationally easy to check whether 0 is a possible value of $f(x)$.

What we are planning to do. Since optimization of set functions is an important practical problem, it is desirable to be able to solve it. In this paper, we will start with a brief mention of the existing methods of solving this problem, then we will explain why these methods are not always applicable and finally, present a new differential formalism that covers previously un-covered practical problems.

In the final section, we will discuss related results and open problems.

\section{The Existing Methods of Optimizing Set Functions: Their Successes (In Brief) and Territorial Division Problem as a Challenge}

\subsection{The Existing Methods of Optimizing Set Functions: A Brief Mention}

The existing methods. There exist many useful differential formalisms for optimizing set functions; an interested reader is referred, e.g., to $[1,2,3,4,5,6$, $7,8,9,10,11,12,13,14,15,16,17,44,45,46,47,48,49,64]$ (this is, of course, not an exhaustive list, set function differentiation is a widely developed area).

Some of these methods are mainly oriented towards the the case when the optimized set has some smoothness, i.e., in practical terms, when we are optimizing a shape that is described by a smooth (or at least piece-wise smooth) surface. For this case, useful differential formalisms have been developed. These formalisms use the fact that small (smooth) deviations from the optimal shape cannot increase the value of the objective function to deduce partial differential equations that describe the optimal shapes.

Other methods reduce set optimization to function optimization, e.g., by replacing a set $A$ by a function $d(x, A)$ that describes the distance from each point $x$ to this set; a more appropriate approach [7] is to use the oriented distance, i.e., $d(x, A)$ when $x \notin A$ and $d(x, C A)$ when $x \in A$ (where $C A$ denotes a complement to the set $A$ ). 
In spite of all usefulness of the existing methods, there are still problems which are not easily covered by these methods. In spite of the numerous successes of the existing methods, there still exist reasonable simple problems that cannot be easily handled by these methods. An example of such problems is the problem of territory division.

The informal meaning of this problem is rather self-explanatory, and in the following text, we will give a precise mathematical description of this problem. For now, we can just mention that the sets that we are interesting in are not necessarily smooth: e.g., in agricultural applications, it often makes sense to, say, plant apple seeds in several disconnected small areas where apples would grow the best; the resulting sets are not smooth, and not connected.

For these and for some other problems, the existing differential formalisms are not directly applicable, and therefore, new differential formalisms are needed. In this paper, we propose a new formalism which solves the territorial division problem.

\subsection{Territorial Division Problem: Towards Formalization}

Let us consider the problem of dividing a piece of territory $a$ between two parties (e.g., the problem of dividing a disputed territory between the two countries).

Comment. This example and the solution given below were first described in $[30,31]$.

This is a problem of finding a set. Each possible division can be uniquely characterized by the set $X$ of all the points that are allocated to the first party.

Indeed, if we know the set $X$ of all the points allocated to the first party, then we can uniquely determined what the second party gets: all the remaining points, i.e., all the points from the complement set $A \backslash X$.

What is the criterion for selecting this set $X$ ?

Towards a criterion for selecting a set: describing the preferences of each party. To describe a reasonable division, we must know, for each possible division of the set $A$ into sets $X$ and $A \backslash X$, how each party evaluates the result of this division.

As a reasonable numerical evaluation of this result for each party, we can take, e.g., the amount of money that this party would be willing to pay for this piece of territory. We will call this amount of money the usefulness of the corresponding piece of territory to this party; we will denote these usefulnesses, correspondingly, by $u_{1}(X)$ and $u_{2}(A \backslash X)$. 
- In reality, we cannot infinitely divide the territory, so, in practice, there are finitely many meaningful pieces (points) into which the territory can be divided. With this in mind, the usefulness $u_{1}(X)$ of the portion $X$ for the first party can be estimated as a sum of usefulnesses of all the points from this set (this is absolutely true, e.g., when we talk about mineral extraction or about the agricultural use of this territory), i.e., as the sum $u_{1}(X)=\sum v_{1}(t)$, where the sum is taken over all points $t \in X$, and $v_{1}(t)$ denotes the usefulness of the point $t$ for the first party.

- The problem with this finitely-many-points approach is that there are many such points, and the corresponding point-by-point computations can take a very long time.

- So, to prevent the long process of enumerating all possible divisions, it makes sense to use a continuous approximation, in which we assume that we can implement an arbitrary division of a planar set $A$ into two sets. In this approximation, the first party's portion is represented by an arbitrary closed set $X \subseteq A$. It is then reasonable to define $u_{1}(X)$ by using a natural continuous analogue of the finite sum, i.e., an integral. In other words, we will assume that $u_{1}(X)=\int_{X} v_{1}(t) d t$.

Similarly, the usefulness $u_{2}(A \backslash X)$ of the piece of territory $A \backslash X$ for the second party is represented as $u_{2}(A \backslash X)=\int_{A \backslash X} v_{2}(t) d t$. It is reasonable to assume that the functions $v_{1}(t)$ and $v_{2}(t)$ are continuous.

The objective function (set function to be optimized). To achieve a reasonable compromise between the interests of both parties, we will use a well-justified notion of Nash's solution, according to which we must choose an alternative for which the product of usefulnesses is the largest possible (see, e.g., $[40,51]$ for description and explanations). In our case, this product is equal to $u_{1}(X) \cdot u_{2}(A \backslash X)$.

Comment. Nash's solution can be easily explained in terms of fuzzy logic (see, e.g., [29, 53]; we are thankful to R. Yager for this explanation): We want both sides to be happy, so we want the first side to be happy and the second side to be happy. We can take $u_{1}(X)$ as the "degree of happiness" of the first side, and $u_{2}(A \backslash X)$ as the "degree of happiness" of the second side. If, in order to formalize "and", we use the operation $d \cdot d^{\prime}$ (one of the two operations originally proposed by L. Zadeh to describe "and"), then the degree to which both sides are satisfied is equal to the product $u_{1}(X) \cdot u_{2}(A \backslash X)$. So, if we look for the division which leads to the largest possible degree of mutual satisfaction, then we must look for the set $X$ for which the product $u_{1}(X) \cdot u_{2}(A \backslash X)$ attains the largest possible value.

So, we arrive at the following formalization of the territory division problem: 


\subsection{Problem Reformulated in Precise Mathematical Terms}

GIVEN: two non-negative continuous functions $v_{1}(t)$ and $v_{2}(t)$ and a bounded open set $A$,

FIND: the closed set $X \subseteq A$ for which

$$
f(X)=u_{1}(X) \cdot u_{2}(A \backslash X) \rightarrow \max ,
$$

where

$$
u_{1}(X)=\int_{X} v_{1}(t) d t \text { and } u_{2}(A \backslash X)=\int_{A \backslash X} v_{2}(t) d t .
$$

Comment. This is a case problem for which the existing methods are not directly applicable and which was a starting point for our new formalism.

\section{A New Differential Formalism for Set Func- tions}

\subsection{Motivations}

The classical result that we are trying to emulate. The use of derivatives in standard optimization is based on the well-known result that if a function $f(x)$, which is defined for all real numbers $x$, attains its (global) maximum (or minimum) at a certain point $x$, then its derivative $f^{\prime}(x)$ at this point $x$ is equal to 0 . Thus, if we know that the function $f(x)$ attains its maximum and minimum, then, to find these maximum and minimum, it is sufficient to find all the points $x$ for which $f^{\prime}(x)=0$, and select the ones for which $f(x)$ is the largest or, correspondingly, the smallest.

Here, the derivative is usually defined as the limit

$$
f^{\prime}(x)=\lim _{h \rightarrow 0, h \neq 0} \frac{f(x+h)-f(x)}{h} .
$$

Practice-motivated reformulation of the classical result. In many practical situations, we have an explicit analytical expression for $f(x)$ and therefore, we can get an analytical expression for the derivative $f^{\prime}(x)$.

However, in many other practical situations, the maximized function is defined only implicitly (e.g., via a partial differential equation), and it is therefore difficult to find an explicit analytical expression for its derivative. In such situations, we can use numerical methods to estimate the desired derivative $f^{\prime}(x)$.

Definition (1) prompts the natural numerical method for computing the derivative: 
- In (1), the derivative is defined as a limit of the ratio $(f(x+h)-f(x)) / h$ when $h$ tends to 0 .

- This means that the smaller $h$, the closer this ratio will be to the desired derivative.

- So, to numerically estimate the derivative, we can take the value of this ratio for some small $h$.

The resulting numerical method often gives a good approximation to the derivative, but this method is definitely not the best, because for smooth functions, its accuracy is $O(h)$, while there exist alternative methods with a much better accuracy $O\left(h^{2}\right)$. To achieve this better accuracy, we can, e.g., take the ratio $(f(x+h)-f(x-h)) /(2 h)$ (this formula, called central difference, is actively used in numerical methods, see, e.g., [23]). With this practical application in mind, it is reasonable to re-define derivative as the limit of the new ratios:

$$
f^{\prime}(x)=\lim _{h \rightarrow 0, h \neq 0} \frac{f(x+h)-f(x-h)}{2 h} .
$$

(This definition is known under the name of Schwartz derivative, see, e.g., [52].)

- From the purely theoretical viewpoint, the new definition is as good as the old one: Namely, one can easily see that if a function $f(x)$ has a derivative in the usual sense, then (2) leads to the same value of the derivative.

The reader should be cautioned that the inverse is not necessarily true: it is possible for a function to have a derivative in the sense of definition (2), but not in the sense of definition (1).

- From the above described practical viewpoint, however, this new definition is better. Indeed:

- for this new definition, the naturally emerging numerical method of computational differentiation is the (asymptotically optimal) $O\left(h^{2}\right)$ one,

- while the numerical method naturally emerging from the original definition (1) has a much worse asymptotic accuracy $O(h)$.

Since the definition (2) is thus more practice-oriented, and since the ultimate goal of our generalization is to be able to solve practical problems, we will try to design an analogue of this practice-oriented definition (2).

Towards the definition of the set derivative. How can we generalize the definition (2) to set functions $f(X)$ ?

For such functions, an analogue of the variable $x$ is a set $X$, and a natural analogue of the small disturbance $h$ is, naturally, also a set $H$. How can reformulate the ratio from (2) in terms of sets? 
- A natural analogue of $x+h$ is the union $X \cup H$, and a natural analogue of the difference $x-h$ is the set difference $X \backslash H$, so we have a natural analogue of the numerator: $f(X \cup H)-f(X \backslash H)$.

- An analogue of the denominator is also pretty much straightforward: of course, we cannot divide by a set, but we can take into consideration that for numbers, $2 h$ is nothing else but the length (1D Lebesgue measure) of the interval $[-h, h]$. So, for an arbitrary set $H$, it is natural to use its Lebesgue measure $\mu_{0}(H)$ as the denominator.

Correspondingly, the condition $h \neq 0$, which made the ratio meaningful by preventing division by 0 , can now be replaced by the similar condition $\mu_{0}(H) \neq$ 0 .

The only remaining problem is how to define the limit. In this definition, it is natural to use the same analogy between intervals and sets as we used to define the appropriate analogue of the denominator.

- In $1 \mathrm{D}$ case, we have defined the limit in which the interval $[-h, h]$ tends to a point (namely, to $\{0\}$ ).

- Therefore, it is reasonable to define a limit for set functions as the limit in which the set $H$ tends to a one-point set, i.e., to a set $\{t\}$ for some point $t$.

To define such a limit, we need a topology on the set of all sets.

A natural topology on this set of all sets is given by Hausdorff metric $d_{\mathcal{H}}(A, B)$, which is defined, for every two sets $A$ and $B$, as the smallest real number $r$ for which $A$ is contained in the $r$-neighborhood of $B$, and $B$ is contained in the $r$-neighborhood of $A: A \subseteq B_{r}(A), B \subseteq B_{r}(A)$.

An $r$-neighborhood of a set $A$ can be defined, e.g., as the set of all points that are $r$-close to some points from $A$, i.e., as $B_{r}(A)=\{t \mid d(t, s) \leq r$ for some $s \in A\}$.

For the topology defined by the Hausdorff metric, $A \rightarrow B$ means that $d_{\mathcal{H}}(A, B) \rightarrow 0$.

Comment. The Hausdorff metric is usually defined only for compact sets $A$ and $B$. If we allow non-compact sets, then, strictly speaking, it is no longer a metric in the topological sense of this word, because we may have $d_{\mathcal{H}}(A, B)=0$ for $A \neq B$; in such a general case, it is only a what is called pre-metric.

We are interested in the case when one of the sets is a one-point set $\{t\}$. In this case, the expression for Hausdorff distance $d_{\mathcal{H}}(H,\{t\})$ can be simplified: this distance is the smallest radius $r$ of the closed ball $B_{r}(\{t\})=\{s \mid d(s, t) \leq r\}$ that contains $H$.

Thus, we arrive at the following definition: 


\subsection{Definitions}

We want the set function to be defined for bounded open and closed subsets of $R^{n}$, and also for finite unions and intersections of such sets:

Definition 1. Let $n \geq 1$ be an integer.

- Let $\mathcal{A}$ be a class of sets that contains all bounded open and closed subsets $X \subseteq R^{n}$ and that is closed under finite union, finite intersection, and set difference. This class of sets will be called an algebra.

- By a set function, we will mean a real-valued function that is defined for all sets from the class $\mathcal{A}$.

\section{Comments.}

- Readers should be aware that we use the word "algebra" just for brevity. For classes of sets, the word algebra is usually reserved for a slightly different notion: when a class is closed under finite unions and complements. If a class $i s$ an algebra in this sense, then it is also closed under finite intersections and set difference; however, the opposite is not necessarily true: e.g., the class of all bounded sets is an algebra in our sense, but it is not closed under complement.

- Definition 1 does not determine the class $\mathcal{A}$ uniquely: e.g., we can take the class $\mathcal{A}$ of all bounded sets, or the class of all Borel sets, or the class of all Lebesgue measurable sets, etc.

Definition 2. Let $f(X)$ be a set function defined on an algebra $\mathcal{A}$, let $X \in \mathcal{A}$ be a set, and let $t \in R^{n}$ be a point. By the derivative of the function $f(X)$ at a set $X$ at a point $t$ we mean the limit

$$
\frac{d f}{d X}(t)=\lim _{H \in \mathcal{A}, H \rightarrow\{t\}, \mu_{0}(H) \neq 0} \frac{f(X \cup H)-f(X \backslash H)}{\mu_{0}(H)}
$$

(where the limit $H \rightarrow\{t\}$ is in the sense of Hausdorff metric).

Comments.

- Our derivative is an analogue of the partial derivative. Namely, if have a function $f\left(x_{1}, \ldots, x_{n}\right)$ of $n$ variables, then, to specify the value of the partial derivative $\partial f / \partial x_{i}\left(x_{1}, \ldots, x_{n}\right)$, we must describe two things:

- first, we must describe the point $x=\left(x_{1}, \ldots, x_{n}\right)$ at which we are computing this derivative;

- second, we must specify the variable $i$ (from 1 to $n$ ) over which we differentiate. 
To provide a similar description for the set derivative, we can take into consideration that an arbitrary subset $X \subseteq R^{n}$ can be uniquely described by its characteristic function $c_{X}(t)$, i.e., by the infinitely many values $\left(c_{X}\left(t_{1}\right), c_{X}\left(t_{2}\right), \ldots\right)$ which correspond to different points $t \in R^{n}$. Thus, to specify the value of the corresponding partial derivative, we must describe two things:

- first, we must describe the point $\left(c_{X}\left(t_{1}\right), c_{X}\left(t_{2}\right), \ldots\right)$ at which we are computing this derivative, i.e., we must specify the set $X$;

- second, we must specify over which of the variables $c_{X}(t)$ we differentiate; variables are in 1-1 correspondence with points $t \in R^{n}$, so specifying a variable is equivalent to specifying a point $t \in R^{n}$.

Thus, to specify a derivative of a set function, we must specify a set $X$ and a point $t$. This is exactly what we are doing.

- Alternatively, for functions of several variables, we can consider, for each point $x=\left(x_{1}, \ldots, x_{n}\right)$, not each partial derivative $\partial f / \partial x_{i}$ by itself, but rather the vector

$$
\nabla f=\left(\frac{\partial f}{\partial x_{1}}, \ldots, \frac{\partial f}{\partial x_{n}}\right)
$$

formed by these derivatives (i.e., a gradient) as the derivative of the function $f\left(x_{1}, \ldots, x_{n}\right)$. Similarly, for a given set $X$, we can consider, as an appropriate definition of a derivative, not the individual values $d f / d X(t)$ taken by themselves, but rather a combination of the values which correspond to different $t \in R^{n}$, i.e., the entire function $t \rightarrow d f / d X(t)$. If we use this definition, then the derivative will no longer be defined as a number, but rather as a function from $R^{n}$ to $R$.

- For additive set functions (i.e., for set functions that are themselves measures), $f(X \cup H)-f(X \backslash H)=f(H)$, and therefore, this definition turns, basically, into the so-called general derivative $D f(t)$ (see, e.g., $[25,27,61,65])$, which is defined as a limit

$$
D f(t)=\lim _{H \in \mathcal{A}, H \rightarrow\{t\}, \mu_{0}(H) \neq 0} \frac{f(H)}{\mu_{0}(H)}
$$

(For additive set functions, the only difference between our definition of a derivative and the generalized derivative is that for a generalized derivative, the requirements on the class $\mathcal{A}$ are slightly different from ours.)

The notion of a general derivative was proposed by Lebesgue himself [38]; Lebesgue has shown that a completely additive set function has a general derivative almost everywhere. It is known that if an additive set function $f(X)$ is absolutely continuous with respect to Lebesgue measure, i.e., if $f(X)=\int_{X} g(t) d t$ for some integrable function $g(t)$, then its 
Radon-Nykodym derivative $g(t)$ is equal almost everywhere to the general derivative $D f(t)$.

- For set functions that are not necessarily additive, similar definitions were proposed in $[59,66]$

- Standard formulas of calculus can be used to describe the derivative of the sum, product, ratio, etc., of two differentiable set functions.

\section{Definition 3.}

- We say that set function $f(X)$ defined on an algebra $\mathcal{A}$ is differentiable if for every set $X \in \mathcal{A}$ and for every point $t \in R^{n}$, it has a derivative.

- We say that a differentiable set function $f(X)$ is continuously differentiable if for every set $X$, the function

$$
\frac{d f}{d X}(t)
$$

is a continuous function of $t$.

\subsection{Notations}

In the following text, we will use the following notation. For every set $A$ :

- $C A$ will denote the complement of the set $A$;

- $\operatorname{Int}(A)$ will denote the interior of the set $A$;

- $\bar{A}$ will denote the closure of the set $A$; and

- $\partial A$ will denote the boundary of the set $A$.

\subsection{Results}

When the set function $f(X)$ is continuously differentiable, we get the direct analogue of the classical relationship between the (global) maxima and minima of a function and the derivative being equal to 0 :

Theorem 1. If a continuously differentiable set function $f(X)$ attains its maximum or its minimum at some set $X$, then

$$
\frac{d f}{d X}(t)=0
$$

for all points $t \in \partial(\operatorname{Int}(X)) \cap \partial(\operatorname{Int}(C X))$. 


\section{Comments.}

- In this theorem and in the following text, whenever we talk about the maximum of a set function, we mean its largest possible value, i.e., its global maximum. Similarly, when we talk about about the minimum of a set function, we mean its smallest possible value, i.e., its global minimum.

We would like to mention, hoewver, that a similar result holds if we are looking for a local maximum or a local minimum of a set function. Here, we say that a set function $f(X)$ attains a local maximum at some set $X$ if there exists an $r$-neighborhood $B_{r}(X)$ of this set $X$ for which $f(X) \geq f(Y)$ for all sets $Y \subseteq B_{r}(X)$.

- In plain words, Theorem 1 says that the derivative of the set function is equal to 0 for all points $t$ from the "border" of the optimal set $X$. In practical terms, we can use this result to find the border of the desired set $X$ and thus, to describe this optimal set itself.

- This theorem is only applicable when both the set $X$ and its complement $A \backslash X$ have interior points. This is a reasonable assumption for problems like territorial division that prompted this research, but, of course, for other problems, this assumption may be false: e.g., the set function $f(X)$ may attain its maximum on a singleton set $X=\{t\}$. To such problems, this approach is not applicable.

- When the set function is simply differentiable, but not necessarily continuously differentiable, then we get inequalities instead of equalities:

Theorem 2. If a differentiable set function $f(X)$ attains its maximum at some set $X$, then:

- for all $t \in \operatorname{Int}(X)$, we have

$$
\frac{d f}{d X}(t) \geq 0
$$

- for all $t \in \operatorname{Int}(C X)$, we have

$$
\frac{d f}{d X}(t) \leq 0 .
$$


Theorem $2^{\prime}$. If a differentiable set function $f(X)$ attains its minimum at some set $X$, then:

- for all $t \in \operatorname{Int}(X)$, we have

$$
\frac{d f}{d X}(t) \leq 0
$$

- for all $t \in \operatorname{Int}(C X)$, we have

$$
\frac{d f}{d X}(t) \geq 0
$$

\subsection{Proofs}

Proving Theorem 2: general idea. Let us start by proving Theorem 2 .

Let $f(x)$ be a differentiable set function that attains its maximum at a set $X$, and let $t \in R^{n}$. According to the definition, the derivative

$$
\frac{d f}{d X}(t)
$$

is equal to the limit of the ratio $(f(X \cup H)-f(X \backslash H)) / \mu_{0}(H)$ when $H \rightarrow\{t\}$ and $\mu_{0}(H) \neq 0$. Let us take, as $H$ 's, a sequence of closed balls $H_{r}=B_{r}(t)$ with a center in $t$ for which $r \rightarrow 0$. Each of these sets $H_{r}$ is a closed bounded set and therefore, $H_{r} \in \mathcal{A}, X \cup H_{r} \in \mathcal{A}$, and $X \backslash H_{r} \in \mathcal{A}$.

According to the above formula for the Hausdorff distance $d_{\mathcal{H}}(X,\{t\})$, the condition $r \rightarrow 0$ implies that $H_{r}=B_{r}(t) \rightarrow\{t\}$; also, we know that $\mu_{0}\left(B_{r}(t)\right) \neq$ 0 for each $r \neq 0$. Therefore, for this sequence of balls, we get

$$
\frac{f\left(X \cup H_{r}\right)-f\left(X \backslash H_{r}\right)}{\mu_{0}\left(H_{r}\right)} \rightarrow \frac{d f}{d X}(t) .
$$

Proving Theorem 2: first part. By definition of an interior point, if $t \in$ Int $(X)$, then we have $B_{r}(t) \subseteq X$ for all sufficiently small $r>0$. Therefore, for such $r$, we have $X \cup H_{r}=X$, and the formula (3) turns into

$$
\frac{f(X)-f\left(X \backslash H_{r}\right)}{\mu_{0}\left(H_{r}\right)} \rightarrow \frac{d f}{d X}(t) .
$$

Since the function $f(X)$ attains its maximum for the set $X$, we have $f(X) \geq$ $f\left(X^{\prime}\right)$ for an arbitrary set $X^{\prime} \in \mathcal{A}$, in particular, for $X^{\prime}=X \backslash H_{r}$. Therefore, the ratio in the left-hand side of the formula (4) is non-negative.

Hence, the limit

$$
\frac{d f}{d X}(t)
$$


of these non-negative ratios should also be non-negative. The first part of Theorem 2 is thus proven.

Proving Theorem 2: second part. Similarly, if $t \in \operatorname{Int}(C X)$, then we have $B_{r}(t) \subseteq C X$ for all sufficiently small $r>0$. Therefore, for such $r, X \backslash H_{r}=X$, and (3) becomes

$$
\frac{f\left(X \cup H_{r}\right)-f(X)}{\mu_{0}\left(H_{r}\right)} \rightarrow \frac{d f}{d X}(t) .
$$

Since the function $f(X)$ attains its maximum for the set $X$, we have $f(X) \geq$ $f\left(X^{\prime}\right)$ for an arbitrary set $X^{\prime} \in \mathcal{A}$, in particular, for $X^{\prime}=X \cup H_{r}$. Therefore, the ratio in the left-hand side of the formula (4) is non-positive and hence, its limit

$$
\frac{d f}{d X}(t)
$$

should also be non-positive. The second part of Theorem 2 is thus also proven, and so is the theorem.

Proving Theorem $2^{\prime}$. This result follows from Theorem 2 if we replace $f$ with $-f$. (Alternatively, it can be proven along the same lines as Theorem 2.)

Proving Theorem 1. Let us now show how Theorem 1 follows from Theorems 2 and $2^{\prime}$. Indeed, let $f(X)$ be a continuously differentiable set function, and let $x$ be a set on which this set function attains its maximum.

Let $t$ be a point from the intersection $\partial(\operatorname{Int}(X)) \cap \partial(\operatorname{Int}(C X))$. Since $t$ belongs to the intersection of these two boundaries, it belongs to each of them.

From $t \in \partial(\operatorname{Int}(X))$, it follows that $t$ is a limit of a sequence of points $t_{m} \in \operatorname{Int}(X)$. For each point $t_{m}$, according to Theorem 2, we have

$$
\frac{d f}{d X}\left(t_{m}\right) \geq 0
$$

By definition of continuous differentiability, the derivative $d f / d X(t)$ is an everywhere defined continuous function of $t$. Thus, from $t_{m} \rightarrow t$, it follows that

$$
\frac{d f}{d X}(t)=\lim \frac{d f}{d X}\left(t_{m}\right)
$$

From (6), we conclude that in the limit,

$$
\frac{d f}{d X}(t) \geq 0
$$

Similarly, from $t \in \partial(\operatorname{Int}(C X))$, it follows that $t$ is a limit of a sequence $t_{m} \in \operatorname{Int}(C X)$. For each point $t_{m}$, according to Theorem 2, we have

$$
\frac{d f}{d X}\left(t_{m}\right) \leq 0
$$


Thus, from $t_{m} \rightarrow t$, it follows that

$$
\frac{d f}{d X}(t)=\lim \frac{d f}{d X}\left(t_{m}\right) .
$$

From (8), we conclude that in the limit,

$$
\frac{d f}{d X}(t) \leq 0 .
$$

So, we have proved the inequalities (7) and (9), according to which the derivative under consideration is both non-negative and non-positive. Thus,

$$
\frac{d f}{d X}(t)=0 .
$$

The theorem is proven.

Comments.

- In the following two sections, we will describe two applications of this new formalism.

- We formulated our theorems for set functions defined on subsets of $R^{n}$, because this is what we need for our applications (see below). However, as one can see from the proof, these results are valid for an arbitrary metric space $M$ with a measure $\mu_{0}$ that is defined on all bounded open and closed sets, and that is positive on all non-degenerate balls (i.e., $\mu_{0}\left(B_{r}(t)\right)>0$ for all $t \in M$ and $r>0$ ).

\section{First Application of the New Formalism: Ter- ritorial Division Problem}

\subsection{Our Results Help to Solve This Problem}

Our formalism is applicable. One can easily check that both functions $u_{1}(X)=\int_{X} v_{1}(t) d t$ and $u_{2}(A \backslash X)=u_{2}(A)-\int_{X} v_{2}(t) d t$ are continuously differentiable, with derivatives correspondingly equal to

$$
\frac{d u_{1}}{d X}(t)=v_{1}(t) \text { and } \frac{d u_{2}}{d X}(t)=-v_{2}(t) .
$$

Therefore, their product, i.e., the set function $f(X)$, is also continuously differentiable, and its derivative is equal to

$$
\frac{d f}{d X}(t)=v_{1}(t) \cdot u_{2}(A \backslash X)-v_{2}(t) \cdot u_{1}(X) .
$$


Let us apply this new formalism. So, if this set function attains its maximum at some set $X$, then, according to Theorem 2, we can make the following two conclusions:

- $v_{1}(t) \cdot u_{2}(A \backslash X)-v_{2}(t) \cdot u_{1}(X) \geq 0$ for all $t \in \operatorname{Int}(X)$;

- $v_{1}(t) \cdot u_{2}(A \backslash X)-v_{2}(t) \cdot u_{1}(X) \leq 0$ for all $t \in \operatorname{Int}(C X)$.

Let us simplify the resulting inequalities. To simplify both inequalities, we can:

- move the negative part to the right-hand side, and then

- divide both sides of the resulting inequality by $v_{2}(t) \cdot u_{2}(A \backslash X)$ (to separate the variables $t$ and $X$ ).

After this transformation, the above conclusions take the following simplified form:

$$
\begin{aligned}
& \frac{v_{1}(t)}{v_{2}(t)} \geq \alpha \text { for all } t \in \operatorname{Int}(X), \\
& \frac{v_{1}(t)}{v_{2}(t)} \leq \alpha \text { for all } t \in \operatorname{Int}(C X),
\end{aligned}
$$

where we denoted

$$
\alpha=\frac{u_{1}(X)}{u_{2}(A \backslash X)} .
$$

Let us be realistic. Sets can be weird and impossible to practically implement: e.g., there is no way to realistically divide the interval $[0,1]$ into the set of all its rational points and the complement set of all its irrational points. So, let us be more realistic, and impose some conditions on the set $X$ to make it more geometric and thus, more realistic in relation to the original problem (of territorial division). Namely, we will assume that:

- $X$ is a closed set, and that

- $X$ is a closure of its own interior.

(These two properties are true for sets bounded by normal smooth curves.) For such sets, we can further simplify the above conclusion.

Using the first realistic assumption. If we are looking for a closed set $X$, then $\operatorname{Int}(C X)=C X$, and (11) takes the form

$$
\frac{v_{1}(t)}{v_{2}(t)} \leq \alpha \text { for all } t \in C X .
$$

Using the second realistic assumption. Similarly, if we are looking for a set $X$ that is the closure of its interior, then, due to continuity of the functions $v_{1}(t)$ and $v_{2}(t)$, we can conclude that $v_{1}(t) / v_{2}(t) \geq \alpha$ for all $t \in X$. 
Let us now combine the consequences of the two realistic assumptions. Thus:

- if $t \in X$, we have $v_{1}(t) / v_{2}(t) \geq \alpha$; and

- if $t \notin X$, we have $v_{1}(t) / v_{2}(t) \leq \alpha$.

Hence:

- If $v_{1}(t) / v_{2}(t)>\alpha$, then we cannot have $t \in X$ and therefore, we must have $t \notin X$.

- Similarly, if $v_{1}(t) / v_{2}(t)<\alpha$, then we cannot have $t \in C X$ and therefore, we must have $t \in X$.

We (almost) got a solution. Thus, if we find the value $\alpha$, then we (almost) have a description of the desired set $X$ :

- this set must contain all the points $t$ for which $v_{1}(t) / v_{2}(t)>\alpha$; and

- this set cannot contain any points $t$ for which $v_{1}(t) / v_{2}(t)<\alpha$.

The only uncertainty is about the points $t$ for which $v_{1}(t) / v_{2}(t)=\alpha$. This uncertainty, however, does not matter much if we assume that for each $\alpha$, the set of all points $t$ for which $v_{1}(t) / v_{2}(t)=\alpha$ has Lebesgue measure 0 , and therefore, whether we add these points to $x$ or to $A \backslash X$ will not affect the usefulnesses $u_{1}(X)$ and $u_{2}(A \backslash X)$.

This assumption is indeed true for generic analytical functions $v_{1}(t)$ and $v_{2}(t)$.

Thus, we can simply define $X$ as, e.g., the set of all the points $t$ for which $v_{1}(t) / v_{2}(t) \geq \alpha$ :

$$
X_{\alpha}=\left\{t \mid \frac{v_{1}(t)}{v_{2}(t)} \geq \alpha\right\} \text {. }
$$

The final step: finding the parameter $\alpha$. If the above "equal to $\alpha$ " uncertainty is thus resolved, then the only remaining problem is how to find the value $\alpha$. Substituting the above expression for $X_{\alpha}$ into the formula (12), we conclude that

$$
\frac{u_{1}\left(X_{\alpha}\right)}{u_{2}\left(A \backslash X_{\alpha}\right)}-\alpha=0
$$

where

$$
u_{1}\left(X_{\alpha}\right)=\int_{\left\{t \mid v_{1}(t) / v_{2}(t) \geq \alpha\right\}} v_{1}(t) d t
$$

and

$$
u_{2}(A \backslash X)=\int_{\left\{t \mid v_{1}(t) / v_{2}(t)<\alpha\right\}} v_{2}(t) d t .
$$

When $\alpha$ increases, then: 
- The set $X_{\alpha}$ become smaller.

- Hence, $u_{1}\left(X_{\alpha}\right)$ become smaller.

- Simultaneously, $A \backslash X_{\alpha}$ becomes larger.

- Because of this, $u_{2}\left(A \backslash X_{\alpha}\right)$ become larger.

- Therefore, the ratio $u_{1}\left(X_{\alpha}\right) / u_{2}\left(A \backslash X_{\alpha}\right)$ decreases as $\alpha$ increases.

- Hence, the difference $u_{1}\left(X_{\alpha}\right) / u_{2}\left(A \backslash X_{\alpha}\right)-\alpha$ also monotonically decreases with $\alpha$.

Hence, we can use, e.g., bisection method to find the value $\alpha$ for which this difference becomes equal to 0 , i.e., to (easily) solve the equation (14).

Comment. From the mathematical viewpoint, our problem is similar to the optimization problem that is well known is statistical hypothesis testing: the so-called Neyman-Pearson Lemma (see, e.g., [28, 39]. In this Lemma, we are given functions $v_{1}(t), \ldots, v_{n}(t), v_{n+1}(t)$, and real numbers $c_{1}, \ldots, c_{n}$, and the problem is: among all the functions $\varphi(t)$ for which $\varphi(t) \in[0,1]$ for all $t$ and $\int \varphi(t) \cdot v_{i}(t) d t=c_{i}$ for $i=1, \ldots, n$, to find the function for which $\int \varphi(t)$. $v_{n+1}(t) d t \rightarrow$ max. It turns out that under reasonable conditions, this maximum is attained when $\varphi(t)$ is a characteristic function of a set $\varphi(t)=c_{X}(t)$ (i.e., the function $\varphi(t)$ takes only values 0 and 1$)$, and for some constants $\alpha_{1}, \ldots, \alpha_{n}$, this set has the following two properties:

- If $v_{n+1}(t)>\alpha_{1} \cdot v_{1}(t)+\ldots+\alpha_{n} \cdot v_{n}(t)$, then $t \in X$.

- If $v_{n+1}(t)<\alpha_{1} \cdot v_{1}(t)+\ldots+\alpha_{n} \cdot v_{n}(t)$, then $t \notin X$.

In the simplest case $n=1$, there is only one value $\alpha_{1}=\alpha$, and these conditions take the following form:

- If $v_{2}(t) / v_{1}(t)>\alpha$, then $t \in X$.

- If $v_{2}(t) / v_{1}(t)<\alpha$, then $t \notin X$.

These are exactly the conditions that we came up with. We can view this as an indirect way of solving our original game theoretic problem. (We are thankful to Ilya Molchanov for this idea.) Indeed, if the the function $f(X)$ can be naturally reformulated in terms of the characteristic function $c_{X}(t)$ : $f(X)=u_{1}(X) \cdot u_{2}(A \backslash X)$, where $u_{1}(X)=\int_{A} c_{X}(t) \cdot v_{1}(t) d t$ and $u_{2}(A \backslash X)=$ $u_{2}(A)-\int_{A} c_{X}(t) \cdot v_{2}(t) d t$, where $u_{2}(A)=\int_{A} v_{2}(t) d t$.

- Instead of considering arbitrary sets $X \subseteq A$, i.e., instead of considering characteristic $\left(\{0,1\}\right.$-valued) functions $c_{X}(t)$, we can consider arbitrary $[0,1]$-valued functions $\varphi(t)$ and try to maximize the product

$$
\left(\int_{A} \varphi(t) \cdot v_{1}(t) d t\right) \cdot\left(u_{2}(A)-\int_{A} \varphi(t) \cdot v_{2}(t) d t\right) .
$$


Since we are increasing the class of possible alternatives, the optimal solution to this problem does not necessarily solve our problem: e.g., if the maximum is attained for some function with $\varphi(t) \in(0,1)$, then this $\varphi(t)$ is not a characteristic function. However, if we are lucky, and if the maximum of the new problem is indeed attained at the characteristic function $c_{X}(t)$ of some set $X$, then this set $X$ is automatically a solution to our original set optimization problem. Let us therefore try our luck.

- If in the new problem the maximum is attained for some function $\varphi(t)$, this means that for the fixed value of the second factor in the product (i.e., for the fixed value of the integral $\int \varphi(t) \cdot v_{2}(t) d t$ ), the first factor $\int \varphi(t) \cdot v_{1}(t) d t$ must take the largest possible value. So, we arrive at an auxiliary conditional optimization problem: maximize $\int \varphi(t) \cdot v_{1}(t) d t$ under the condition that $\int \varphi(t) \cdot v_{2}(t) d t=c_{2}$ for some real number $c_{2}$.

- From the Neyman-Pearson Lemma, it follows that the solution to this auxiliary problem is indeed attained for a characteristic function $\varphi(t)=$ $c_{X}(t)$, so we can indeed conclude that the corresponding set $X$ is indeed the solution to our original territorial division problem.

Does this mean that we do not need our formalism, and we can always solve solve the problem of optimizing set functions $f(X)$ by extending them to characteristic functions $c_{X}(t)$ to arbitrary functions $\varphi(t)$ ? Not necessarily:

- First of all, this approach (expending from characteristic functions to arbitrary functions) may not always work. It was our luck that we ended up with a characteristic function as an optimal solution. In general, the maximum may be attained on a function that takes values from $(0,1)$. Neyman-Pearson Lemma is about optimizing a linear function under linear constraints, and our main purpose is to deal with non-linear functions.

- Even when this approach does work, it is a very roundabout way to optimize a function $f(X)$ of a set variable $X$. Our approach - differentiating this function $f(X)$ with respect to $X$ and equating the derivative to $0-$ is much more straightforward and natural.

\subsection{Comment 1: A More Complicated Utility Function}

A more realistic formulation of the territorial division problem: informal explanation. In the above text, we measured usefulness by the equivalent money amount. A more realistic approach would be to assume that the usefulness (normally called utility in game theory) is actually a monotonic function of the money amount [40,51]. 
Towards mathematical formalization of this more realistic formulation. In this case, the problem becomes slightly more complicated:

- instead of maximizing the product $u_{1}(X) \cdot u_{2}(A \backslash X)$ of the two integrals,

- we have to maximize the product $f(X)=m_{1}\left(u_{1}(X)\right) \cdot m_{2}\left(u_{2}(A \backslash X)\right)$, where:

- $u_{1}(X)$ and $u_{2}(A \backslash X)$ are the same integrals as before, and

- $m_{1}(z)$ and $m_{2}(z)$ are strictly increasing functions.

Comments.

- Since we want to use differentiation to find the maximum, we will assume that these functions $m_{i}(z)$ are differentiable.

- The case when $m_{1}(z)=m_{2}(z)$ can be naturally interpreted in terms of fuzzy logic [29, 53]. Indeed, a very general class of "and"-operations (called t-norms) consists of the so-called strict Archimedean t-norms, i.e., operations of the type $d \& d^{\prime}=\psi^{-1}\left(\psi(d) \cdot \psi\left(d^{\prime}\right)\right)$ for some monotonic function $\psi(z)$. Thus, if $u_{1}(X)$ is the "degree of happiness" of the first side, and $u_{2}(A \backslash X)$ is the "degree of happiness" of the second side, then the degree to which the first side is happy and the second side is happy is equal to $u_{1}(X) \& u_{2}(A \backslash X)=\psi^{-1}\left(\psi\left(u_{1}(X)\right) \cdot \psi\left(u_{2}(A \backslash X)\right)\right)$. If we look for the division which leads to the largest possible degree of mutual satisfaction, then we must look for the set $X$ for which this expression $u_{1}(X) \& u_{2}(A \backslash X)$ attains the largest possible value. Since the function $\psi(z)$ is monotonically increasing, this expression attains the largest possible value if and only if the product $\psi\left(u_{1}(X)\right) \cdot \psi\left(u_{2}(A \backslash X)\right)$ attains the largest possible value. This is exactly the above generalized Nash's expression for $m_{1}(z)=m_{2}(z)=\psi(z)$.

Our formalism is applicable to this new problem as well. Differentiating the new expression with respect to $X$, we can also conclude that there exists a value $\alpha$ for which the desired set $X$ satisfies the following properties:

- the set $X$ contains all the points $t$ for which $v_{1}(t) / v_{2}(t)>\alpha$; and

- the set $X$ does not contain any points $t$ for which $v_{1}(t) / v_{2}(t)<\alpha$.

Substituting the resulting expression for $X_{\alpha}$ into the corresponding formula for $\alpha$, we get a slightly more complicated formula for determining $\alpha$, but the monotonicity property still stands for this new formula, and thus, we can still use bisection to find the desired value $\alpha$.

Comment. The solutions described in this and in the following subsection are not very complicated: they use standard arguments well known in calculus to solve the corresponding problems of optimizing set functions. This simplicity is 
what we wanted to achieve in the first place, by describing differentiation of set functions as a natural generalization of the standard derivative from calculus. This simplicity is what we are proud of.

\subsection{Comment 2: A More General Territorial Division Problem}

Informal explanation of the new problem. It is natural to consider a more general territorial division problem, in which more than two parties want to divide the territory.

Mathematical formalization of the new problem. In the simplest formulation, we want to find the sets $X_{1}, \ldots, X_{k}$ such that:

- $X_{i} \cap X_{j}=\emptyset$ for $i \neq j$,

- $\cup X_{i}=A$, and

- $u_{1}\left(X_{1}\right) \cdot u_{2}\left(X_{2}\right) \cdot \ldots \cdot u_{n}\left(X_{n}\right) \rightarrow \max$, where $u_{i}\left(X_{i}\right)=\int_{X_{i}} v_{i}(t) d t$ for some continuous functions $v_{i}(t)$.

Comment. This formulation corresponds to applying the original Zadeh's "and" $d \& d^{\prime}=d \cdot d^{\prime}$ to the degree of happiness of several participants.

For this new problem, we cannot use our formalism directly. This more general problem does not directly fall into our scheme, because:

- our theorems describe unconditional optimization of set functions, while

- in this new multi-party problem, we need to optimize a set function under the conditions $\left(\cup X_{i}=A\right.$ and $\left.X_{i} \cap X_{j}=\emptyset\right)$.

However, we can still use our theorems indirectly and solve this problem.

Our main idea of solving this problem. Our main idea here is similar to the way how we can use differentiation of functions of one variable to optimize function of several variables: if we know that a function $f\left(x_{1}, \ldots, x_{n}\right)$ of several (numerical) variables attains its maximum for some tuple $\left(x_{1}, \ldots, x_{n}\right)$, then, in particular, we can fix the values of all these variables except for $x_{1}$, and conclude that the resulting function of a single variable should also attain its maximum. Therefore, for the optimal value $x_{1}$, the derivative of that resulting function of one variable is equal to 0 . This derivative is exactly the partial derivative of $f$ with respect to $x_{1}$. We can then repeat the same construction for all other variables.

Let us apply this idea. Let us apply a similar idea to the territorial division problem. Let us assume that $X_{1}, \ldots, X_{k}$ form the optimal division, i.e., the division for which the product

$$
u_{1}\left(X_{1}\right) \cdot u_{2}\left(X_{2}\right) \cdot \ldots \cdot u_{k}\left(X_{k}\right)
$$

takes the largest possible value. To find $X_{i}$, we will fix some of these sets $X_{i}$. 
- For the function of several variables, we fixed the values of all the variables but one.

- For the territorial division problem, we cannot fix all the sets but one, because if we do that (i.e., if we describe what each party gets, except for one party), then the remaining set will be uniquely determined: the remaining party gets exactly all the points that nobody else got.

Since we cannot fix all the sets but one, we will do the next best thing: we will fix all the allocations but two.

Reducing the new problem (of dividing between more than two parties) to the old one (of division between two parties). For example, we can fix the sets $X_{3}, X_{4}, \ldots, X_{k}$, and change $X_{1}$ and $X_{2}$. Since all allocations $X_{3}, X_{4}, \ldots$, are fixed, we thus fix the set $A^{\prime}=X_{1} \cup X_{2}$ of not allocated points; if we select $X_{1}$, then we can uniquely determine $X_{2}$ as $X_{2}=A^{\prime} \backslash X_{1}$. So, we do get a problem with a single set. What is the objective function for this problem?

Since $X_{3}, X_{4}, \ldots$ are fixed, the original objective function

$$
u_{1}\left(X_{1}\right) \cdot u_{2}\left(X_{2}\right) \cdot u_{3}\left(X_{3}\right) \cdot \ldots \cdot u_{k}\left(X_{k}\right)
$$

attains its largest possible value if and only the product of the first two terms is the largest possible: $u_{1}\left(X_{1}\right) \cdot u_{2}\left(X_{2}\right) \rightarrow$ max. So, we conclude that we must select $X_{1} \subseteq A^{\prime}$ from the condition that $u_{1}\left(X_{1}\right) \cdot u_{2}\left(A^{\prime} \backslash X_{2}\right) \rightarrow$ max.

Let us use the known solution of the old problem to solve the new one. This is exactly the problem that we have been solving before, and we know its solution: there exist some value $\alpha_{12}\left(=u_{1}\left(X_{1}\right) / u_{2}\left(X_{2}\right)\right)$, such that the borderline between the points allocated to the first party and the points allocated to the second party is where $v_{1}(t) / v_{2}(t)=\alpha_{12}$. In other words:

- if $v_{1}(t) / v_{2}(t)>u_{1}\left(X_{1}\right) / u_{2}\left(X_{2}\right)$ (i.e., if $\left.v_{1}(t) / u_{1}\left(X_{1}\right)>v_{2}(t) / u_{2}\left(X_{2}\right)\right)$, then $t$ is allocated to $X_{1}$;

- if $v_{1}(t) / v_{2}(t)<u_{1}\left(X_{1}\right) / x_{2}\left(x_{2}\right)$ (i.e., if $\left.v_{1}(t) / u_{1}\left(X_{1}\right)<v_{2}(t) / u_{2}\left(X_{2}\right)\right)$, then $t$ is allocated to $X_{2}$.

Similarly, by considering other pairs of parties, we can conclude that for each $i$ and $j$, the borderline between $x_{i}$ and $x_{j}$ lies where $v_{i}(t) / v_{j}(t)=u_{i}\left(X_{i}\right) / u_{j}\left(X_{j}\right)$, i.e.:

- if $v_{i}(t) / u_{i}\left(X_{i}\right)>v_{j}(t) / u_{j}\left(X_{j}\right)$, then $t$ is allocated to $X_{i}$;

- if $v_{i}(t) / u_{i}\left(X_{i}\right)<v_{j}(t) / u_{j}\left(X_{j}\right)$, then $t$ is allocated to $X_{j}$.

In general, a point $t$ is allocated to $X_{i}$ if for every other set $X_{j}$, we have $v_{i}(t) / u_{i}\left(X_{i}\right) \geq v_{j}(t) / u_{j}\left(X_{j}\right)$, i.e., if the ratio $v_{i}(t) / u_{i}\left(X_{i}\right)$ is the largest of $k$ ratios $v_{1}(t) / u_{1}\left(X_{1}\right), \ldots, v_{k}(t) / u_{k}\left(X_{k}\right)$. 
Conclusion: solution to the new problem. If we denote $u_{i}\left(X_{i}\right)$ by $\alpha_{i}$, then we can describe this solution as follows: every point $t$ is allocated to the set $X_{i}$ for which

$$
\frac{v_{i}(t)}{\alpha_{i}}=\max _{j=1, \ldots, k} \frac{v_{j}(t)}{\alpha_{j}} .
$$

The values $\alpha_{i}$ can be then determined from the condition that

$$
u_{i}\left(X_{i}\right)=\int_{X_{i}} v_{i}(t) d t=\alpha_{i} .
$$

Thus, we get $k$ equations with $k$ unknowns $\alpha_{1}, \ldots, \alpha_{k}$ to determine these parameters $\alpha_{i}$.

What if usefulness is a monotonic function of money values. A similar solution (15) holds if we assume that usefulness is a monotonic function of the money value, i.e., if we want to maximize the expression

$$
m_{1}\left(u_{1}\left(X_{1}\right)\right) \cdot m_{2}\left(u_{2}\left(X_{2}\right)\right) \cdot \ldots \cdot m_{k}\left(u_{k}\left(X_{k}\right)\right)
$$

the only difference is that the equations for $\alpha_{i}$ will be, in this case, more complicated.

Comment. If the functions $v_{i}(t)$ are not necessarily continuous, but only Lebesgue measurable, then the set function is differentiable for almost all $t$, and similar results follow modulo set of measure 0 .

\section{Second Application of the New Formalism: Statistical Example - Excess Mass Method}

\subsection{Excess Mass Method: the Main Idea}

In $[26,50]$, it was shown that for a probability measure on $R^{n}$ with density $\rho(t)$, and for each level $\alpha$, the $\alpha$-level contour $X_{\alpha}=\{t \mid \rho(t) \geq \alpha\}$ can be alternatively defined as a set that maximizes the functional

$$
\varepsilon(X)=\mu(X)-\alpha \cdot \mu_{0}(X),
$$

where:

- $\mu(X)=\int_{X} \rho(t) d t$ is the probability of $t$ being in the set $X$, and

- $\mu_{0}(X)\left(=\int_{X} d t\right)$ is the Lebesgue measure of this set $X$.

This functional $\varepsilon(X)$ is called the excess mass.

The excess mass method is very useful: the extremal representation of $\alpha$ contours has lead to several efficient statistical algorithms; see, e.g., [26, 50, 54, $60]$. 


\subsection{The Basic Result of Excess Mass Method Follows from Our Theorems}

Our formalism is applicable. Let us assume, for simplicity, that $\rho(t)$ is a continuous function. Then, one can easily check that the excess mass set function $\varepsilon(X)$ is differentiable, and its derivative is equal to

$$
\frac{d \varepsilon}{d X}(t)=\rho(t)-\alpha
$$

The result of applying our formalism. So, if this set function attains its maximum at some set $X$, then, according to Theorem 2, we can make two conclusions:

$$
\begin{gathered}
\frac{d \varepsilon}{d X}(t)=\rho(t)-\alpha \geq 0 \text { for all } t \in \operatorname{Int}(X) ; \\
\frac{d \varepsilon}{d X}(t)=\rho(t)-\alpha \leq 0 \text { for all } t \in \operatorname{Int}(C X) .
\end{gathered}
$$

Looking for a realistic set $X$. Similar to the first application of our formalism - to game theory - we can restrict ourselves to realistic sets $X$, i.e., to sets $X$ that are closed and that coincide with the closure of their interior.

If we are looking for a closed set $X$, then $\operatorname{Int}(C X)=C X$, and (17) takes the form

$$
\frac{d \varepsilon}{d X}(t)=\rho(t)-\alpha \leq 0 \text { for all } t \in C X .
$$

Similarly, if we are looking for a set $X$ that is the closure of its interior, then, due to continuity of the function $\rho(t)$, we can conclude that $\rho(t)-\alpha \geq 0$ for all $t \in X$. Thus:

- if $t \in X$, we have $\rho(t) \geq \alpha$; and

- if $t \notin X$, we have $\rho(t) \leq \alpha$.

Hence:

- If $\rho(t)>\alpha$, then we cannot have $t \in X$ and therefore, we must have $t \notin X$.

- Similarly, if $\rho(t)<\alpha$, then we cannot have $t \in C X$ and therefore, we must have $t \in X$.

Thus, we (almost) have a description of the desired set $X$ :

- this set must contain all the points $t$ for which $\rho(t)>\alpha$; and

- this set cannot contain any points $t$ for which $\rho(t)<\alpha$. 
Conclusion. Thus, we can conclude that the desired set $X$ is almost equal to the $\alpha$-contour $X_{\alpha}=\{t \mid \rho(t) \geq \alpha\}$, almost equal in the following sense:

- $X$ does contain all the points for which $\rho(t)$ is strictly larger than $\alpha$;

- $X$ does not contain any points that are not in the $\alpha$-contour (i.e., for which $\rho(t)<\alpha)$; but

- $X$ may miss some of the points $t$ for which $\rho(t)$ is exactly equal to $\alpha$.

\section{Further Directions, Related Results, and Open Problems}

\subsection{Limitations of the Above Differential Formalism}

Similarly to the standard differentiation, all this formalism provides is an equation that is a necessary condition for the optimum. In some cases, this equation directly leads to a solution, but in some other cases, this equation is, itself, difficult to solve.

\subsection{Gradient-Type Iterative Methods}

Iterative methods are needed. Since it is sometimes difficult to directly solve an equation obtained by equating the derivative to 0 , it is desirable to design iterative gradient-type methods for optimizing set functions.

Example of a gradient-type method for optimizing set functions. For the case of a finite universal set $U$, such a method has been proposed by M. Zakharevich in [68] (see also [32, 33, 36]). We have already mentioned that for this case, an arbitrary set $X \subseteq U$ can be described as a sequence of binary variables $x_{1}, \ldots, x_{n} \in\{0,1\}$ where $x_{i}$ is equal to 1 or 0 depending on whether the $i$-th element of the universal set belongs to the set $X$ or not. Since each variable $x_{i}$ has only two possible values, as a "derivative" of a function $f\left(x_{1}, \ldots, x_{n}\right)$ relative to $x_{i}$, we take the difference between the value of this function for $x_{1}=1$ and $x_{i}=0$, i.e., the difference $f\left(x_{1}, \ldots, x_{i-1}, 1, x_{i+1}, \ldots, x_{n}\right)-f\left(x_{1}, \ldots, x_{i-1}, 0, x_{i+1}, \ldots, x_{n}\right)$.

By using this definition, we overcame the problem of defining the derivative, but we still need to overcome another problem become we get a working iterative method:

- the values of all the variables should always be 0 or 1 , but

- with thus defined "gradient" $D f_{i}$, the new value $x_{i}^{\prime}=x_{i}-\lambda \cdot D f_{i}$ is not necessarily equal to 0 or 1. 
To overcome this second problem (and thus get an iterative method), we allow arbitrary real numbers on the intermediate stages of the iterative process, and only after the iterations stop, we transform these values back to the set $\{0,1\}$ : e.g., we can use a threshold $t$ and set $x_{i}$ to 1 if the corresponding real value was $\geq t$, and set $x_{i}$ to 0 otherwise.

This method is reasonably successful. The resulting algorithm turned out to be reasonably successful for solving several known NP-hard problems, including the so-called propositional satisfiability problem (historically the first NP-hard problem).

This gradient-type method turns out to be related to neural, fuzzy, etc. Interestingly, the resulting algorithm coincides with a heuristic method proposed by S. Maslov in the 1980s [41, 42, 43] as a way of simulating biological neurons (see also $[32,33,34,36,63]$ ).

This method was originally proposed based on the idea of simulating biological neurons, but it later turned out that exactly these same formulas follow:

- from fuzzy logic heuristics,

- from the ideas of chemical computing (i.e., simulating chemical reactions),

- from heuristics of numerical optimization approach,

- from the ideas of freedom of choice, etc.

(for a survey and latest results, see $[33,36]$ ).

\subsection{What If Iterative Methods Do Not Work Either?}

In many practical cases, either the differential approach work directly, or at least derivative-based iterative methods work. However, in other practical cases, none of these methods work. How can we then solve the problem of optimizing a given set function?

\subsection{Considering Finite-Parametric Families of Sets}

The main reason why set optimization problems are so difficult is, as we have mentioned, the fact that for these problems, the class of all alternatives (sets) is enormous.

If the set of alternatives can be characterized by finitely many parameters $x_{1}, \ldots, x_{n}$, i.e., if this class is finite-dimensional (in the topological meaning of the word dimension), then finding the optimal values of these parameters $x_{i}$ is a doable problem. However, if alternatives are sets, even convex compact sets in $R^{2}$, then get a more complicated infinite-dimensional optimization problem. Indeed, if we only consider triangles, then we get a family of sets which is characterized by 6 parameters ( 2 coordinates of each of 3 vertices), so we get a 
6-dimensional family of sets. Every convex set can be crudely approximated by a triangle, but if we want a better approximation, we must use polygons with more vertices. For every $n$, to describe an $n$-gon, we need $2 n$ parameters, so the family of all $n$-gons is ( $2 n)$-dimensional. The family of all possible convex compact sets include all these families of increasing dimension and is, therefore, infinite-dimensional.

The more vertices we use, the better the approximation. So, if we cannot solve the infinite-dimensional optimization problem, it is natural to restrict ourselves to a finite-dimensional (finite-parametric) family of sets which provides a good enough approximation. These approximating sets are not necessarily polygons: we may take other families of convex sets. With this restriction, the objective function becomes a function of finitely many parameters, and thus, we can use known optimization techniques, for solving the corresponding optimization problem.

\subsection{A Related Problem - Choosing the Optimal Family of Sets: Successes and Open Problems}

\section{The problem.}

- At first glance, it may seem that with the idea of restricting ourselves to a finite-parametric family, we overcame the difficulties of optimizing set functions.

- However, if we seriously follow this approach, then the natural next question is:

Which of the many finite-parametric families should we choose?

In other words, instead of the problem of finding the most appropriate set a (i.e., a set for which the objective function takes the largest possible value), we now face an even more complicated problem: to find the most appropriate family of sets (i.e., family for which the corresponding objective function has the best possible value).

Successes: in some cases, this problem is solvable. There are cases when this complicated problem can be solved: e.g., when we know that the corresponding problem is invariant with respect to natural symmetries. In this case, if the optimum is achieved for a single family, this family must also be invariant with respect to the same symmetries. This invariance often enables us to determine the optimal family:

- In processing astronomical images, this approach not only leads to the explicit expressions for optimal families, but it also explains the families of image sets that are traditional used in astronomy, such as spirals, etc. $[19,20,21,55]$. 
- In numerical mathematics, this approach explains, e.g., the successful use of ellipsoids in modern numerical methods [19]: under reasonable symmetry conditions, ellipsoids turn out to be the best family for representing uncertainty.

- In non-destructive testing of aerospace objects, we get the optimal families of sets where we should place sensors $[55,56]$.

Remaining problems. These successes do not mean that this symmetry approach always works: in some practical problems, we do not have reasonable symmetries, and therefore, this approach is not applicable. It is thus desirable to extend the above differential formalism for finding the optimal set so that the extended formalism will provide us with optimal families of sets. Developing such a generalized formalism is, therefore, an important open problem.

Acknowledgments. This work was partially supported by NASA under cooperative agreement NCCW-0089, by NSF under grants No. DUE-9750858 and EEC-9322370, and by the Future Aerospace Science and Technology Program (FAST) Center for Structural Integrity of Aerospace Systems, effort sponsored by the Air Force Office of Scientific Research, Air Force Materiel Command, USAF, under grant number F49620-95-1-0518.

The authors are very thankful to Jean-Pierre Aubin, Ilya Molchanov, and Ronald R. Yager for important references and advise.

\section{References}

[1] J.-P. Aubin, Mutational and Morphological Analysis, to appear.

[2] M. C. Delfour and J.-P. Zolesio, "Shape sensitivity analysis via a penalization method", Annali di Matematica pura ed applicata (IV), 1988, Vol. 151, pp. $179-212$.

[3] M. C. Delfour and J.-P. Zolesio, "Shape sensitivity" analysis via min max differentiability", SIAM J. Control and Optimization, 1988, Vol. 26, No. 4, pp. 834-862.

[4] M. C. Delfour and J.-P. Zolesio, "Analyse des problèmes de forme par la dérivation des minimax", In: H. Attouch, J.-P. Aubin, F. Clarke, and I. Ekeland, Eds., Analyse Non Linéaire, Gauthier-Villars \& C.R.M. Université de Montréal, 1989, pp. 211-228.

[5] M. C. Delfour and J.-P. Zolesio, "Sensitivity method and Lagrangian formulation for the computation of the shape Hessian", SIAM J. Control and Optimization, 1991, Vol. 24, No. 5, pp. 835-883. 
[6] M. C. Delfour and J.-P. Zolesio, "Functional analytic methods in shape analysis", Proc. IFIP Workshop on Boundary Control and Boundary Variation, Sophia Antipolis, June 1992.

[7] M. C. Delfour and J.-P. Zolesio, "Shape analysis via oriented distance functions", Journal of Functional Analysis, 1994, Vol. 123, pp. 129-201.

[8] M. C. Delfour and J.-P. Zolesio, "A boundary differential equation for thin shells", J. Diff. Equations, 1995, Vol. 119, pp. 426-449.

[9] M. C. Delfour and J.-P. Zolesio, "Tangential differential equations for dynamical thin/shallow shells", J. Diff. Equations, 1996, Vol. 128, pp. 125167.

[10] M. C. Delfour and J.-P. Zolesio, "Structure of shape derivatives for domains nonsmooth domains", J. Functional Analysis, to appear.

[11] M. C. Delfour and J.-P. Zolesio, Intrinsic differential geometry and theory of thin shells, Scuola Normale di Pisa, to appear.

[12] L. Doyen, Optimisation, évolution et contrôle de formes, Thèse de l'Université de Paris-Dauphine, 1993.

[13] L. Doyen, "Filippov and invariance theorems for mutational inclusions for tubes", Set-Valued Analysis, 1993, Vol. 1, pp. 289-303.

[14] L. Doyen, "Inverse function theorems and shape optimization", SIAM Journal on Control and optimization, 1994, Vol. 32, No. 6, pp. 1621-1642.

[15] L. Doyen, "Mutational equations for tubes and vision based control", Journal of Mathematical Imaging and Vision, to appear.

[16] L. Doyen, "Shape Lyapunov functions", J. Math. Anal. Appl., to appear.

[17] L. Doyen, "Lagrangian multipliers in Shape Optimization under shape constraints", Proceedings of IFIP Workshop Boundary Control, Centre de Mathématiques Appliquées, Ecole des Mines, Sophia-Antipolis, to appear.

[18] R. P. Feynman, R. B. Leighton, and M. L. Sands, The Feynman Lectures On Physics, Addison-Wesley, Redwood City, CA, 1989.

[19] A. Finkelstein, O. Kosheleva, and V. Kreinovich, "Astrogeometry, error estimation, and other applications of set-valued analysis", ACM SIGNUM Newsletter, 1996, Vol. 31, No. 4, pp. 3-25.

[20] A. Finkelstein, O. Kosheleva, and V. Kreinovich, "Astrogeometry: towards mathematical foundations", International Journal of Theoretical Physics, 1997, Vol. 36, No. 4, pp. 1009-1020. 
[21] A. Finkelstein, O. Kosheleva, and V. Kreinovich, "Astrogeometry: geometry explains shapes of celestial bodies", Geombinatorics, 1997, Vol. VI, No. 4, pp. $125-139$.

[22] M. Garey and D. Johnson, Computers and intractability: a guide to the theory of NP-completeness, Freeman, San Francisco, 1979.

[23] C. F. Gerald and P. O. Wheatley, Applied Numerical Analysis, AddisonWesley, Reading, MA, 1992.

[24] J. Goutsias, R. P. S. Mahler, and H. T. Nguyen (eds.), Random Sets: Theory and Applications, Springer-Verlag, N.Y., 1997.

[25] H. Hahn and A. Rosenthal, Set functions, The University of New Mexico Press, Albuquerque, NM, 1948.

[26] J. A. Hartigan, "Estimation of a convex density contour in two dimensions", Journal of the American Statistical Association, 1987, Vol. 82, No. 397, pp. $267-270$.

[27] C. A. Hayes and C. Y. Pauc, Derivation and martingales, Springer-Verlag, NY, 1970.

[28] M. G. Kendall and A. Stuart, The advanced theory of statistics, Griffin, 1961.

[29] G. Klir and B. Yuan, Fuzzy sets and fuzzy logic: theory and applications. Prentice Hall, Upper Saddle River, NJ, 1995.

[30] O. M. Kosheleva and V. Kreinovich, On the optimal territory division, Technical Report, Leningrad Technological Center "Informatika", Leningrad, 1989 (in Russian).

[31] V. Kreinovich, "Nash's solution for the territory division problem", Proceedings of the 3rd USSR National Conference on Game Theory, Odessa, 1974, pp. 117-118 (in Russian).

[32] V. Kreinovich, "Semantics of S. Yu. Maslov's iterative method," In: Problems of Cybernetics, Moscow, 1987, Vol. 131, pp. 30-62 (in Russian); English translation in: V. Kreinovich and G. Mints (eds.), Problems of reducing the exhaustive search, American Mathematical Society, Providence, RI, 1997, pp. 23-52.

[33] V. Kreinovich, "S. Maslov's Iterative Method: 15 Years Later (Freedom of Choice, Neural Networks, Numerical Optimization, Uncertainty Reasoning, and Chemical Computing)", In: V. Kreinovich and G. Mints, eds. Problems of reducing the exhaustive search, American Mathematical Society, Providence, RI, 1997, pp. 175-189. 
[34] V. Kreinovich and L. O. Fuentes, "Simulation of chemical kinetics - a promising approach to inference engines," in: J. Liebowitz (ed.), Proceedings of the World Congress on Expert Systems, Orlando, Florida, 1991, Pergamon Press, N.Y., Vol. 3, pp. 1510-1517.

[35] V. Kreinovich, A. Lakeyev, J. Rohn, and P. Kahl, Computational complexity and feasibility of data processing and interval computations, Kluwer, Dordrecht, 1997.

[36] V. Kreinovich and G. Mints, eds., Problems of reducing the exhaustive search. American Mathematical Society, Providence, RI, 1997.

[37] L. D. Landau and E. M. Lifschitz, The Classical Theory of Fields, Butterworth-Heinemanm, Oxford, UK, 1987.

[38] H. Lebesgue, Leçons sur l'integration et la recherche des fonctions primitives, Gauthier-Villars, Paris, 1904 (2nd ed., 1928).

[39] E. L. Lehmann, Testing statistical hypotheses, Wiley, N.Y., 1959.

[40] D. R. Luce and H. Raiffa, Games and Decisions, Introduction and critical survey, John Wiley \& Sons, Inc., New York, 1957.

[41] S. Yu. Maslov, "Iterative methods in intractable problems as a model of intuitive methods", Abstracts of the 9th All-Union Symposium on Cybernetics, 1981, pp. 52-56 (in Russian).

[42] S. Yu. Maslov, "Asymmetry of cognitive mechanisms and its implications", Semiotika i Informatika, 1983, Vol. 20, pp. 3-31 (in Russian).

[43] S. Yu. Maslov, Theory of deductive systems and its applications, MIT Press, Cambridge, MA, 1987.

[44] J. Mattioli, "Differential Inclusions for Mathematical Morphology", In SPIE: Application of Artificial Neural Network, San Diego, July 11-16, 1993.

[45] J. Mattioli, Problèmes inverses et relations différentielles en morphologie mathématique, Thèse de l' Université de Paris-Dauphine, 1993.

[46] J. Mattioli, "Differential Relations of Morphological Operators", In Proceedings of the Conference on Mathematical Morphology and its applications to Signal Processing, Barcelona, Spain, May 12-14, 1993.

[47] J. Mattioli, "Relations Différentielles d'Opérations de la Morphologie Mathématique", Revue Technique Thomson, 1993, Vol. 25, No. 1, pp. 281303 
[48] J. Mattioli, "Relations différentielles d'opérations de la morphologie mathématique", C.R. Acad. Sci. Paris, 1993, Vol. 316, pp. 879-884.

[49] J. Mattioli, "Minkowski operations and vector spaces", Set-Valued Analysis, to appear.

[50] D. W. Müller and G. Sawitzki, Using excess mass estimates to investigate the modality of a distribution, University of Heidelberg, Preprint No. 398, SFB 123.

[51] R. B. Myerson, Game theory. Analysis of conflict, Harvard University Press, Cambridge, MA, 1991.

[52] I. P. Natanson, Theory of functions of a real variable, F. Ungar Publ., N.Y., 1961.

[53] H. T. Nguyen and E. A. Walker, A first course in fuzzy logic, CRC Press, Boca Raton, Florida, 1997.

[54] D. Nolan, "The excess-mass ellipsoid", Journal of Multivariate Analysis, 1991, Vol. 39, pp. 348-371.

[55] R. Osegueda, C. Ferregut, M. J. George, J. M. Gutierrez, and V. Kreinovich, "Non-Equilibrium Thermodynamics Explains Semiotic Shapes: Applications to Astronomy and to Non-Destructive Testing of Aerospace Systems", Proceedings of the International Conference on Intelligent Systems and Semiotics (ISAS'97), National Institute of Standards and Technology Publ., Gaithersburg, MD, 1997, pp. 378-382.

[56] R. Osegueda, C. Ferregut, M. J. George, J. M. Gutierrez, and V. Kreinovich, "Computational geometry and artificial neural networks: a hybrid approach to optimal sensor placement for aerospace NDE", In: C. Ferregut, R. Osegueda, and A. Nuñez (eds.), Proceedings of the International Workshop on Intelligent NDE Sciences for Aging and Futuristic Aircraft, El Paso, TX, September 30-October 2, 1997, pp. 59-71.

[57] C. H. Papadimitriou, Computational Complexity, Addison Wesley, San Diego, 1994.

[58] P. M. Pardalos, Complexity in Numerical Optimization, World Scientific, Singapore, 1993.

[59] C. Petrescu-Prodan, "On the derivative of a set function", Studii si Cercetari Matematica, 1992, Vol. 4,, No. 3, pp. 253-259.

[60] W. Polonik, "Measuring mass concentrations and estimating density contour clusters - an excess mass approach", The Annals of Statistics, 1995, Vol. 23, No. 3, pp. 855-881. 
[61] G. E. Shilov and B. L. Gurevich, Integral, measure, and derivative: a unified approach, Dover, N.Y., 1977.

[62] V. I. Sinyansky, A. A. Stotsky, and V. Ya. Kreinovich, "Reconstructing the field phase on the radio telescope aperture from the amplitude values on the focal point and on the aperture", In: Radioastronomical Hardware. Antennas and Methods. Proceedings of the XIV USSR National Radioastronomical Conference, October 28-30, 1982, Armenian Academy of Sciences, Erevan, pp. 246-247 (in Russian).

[63] O. Sirisaengtaksin, L. O. Fuentes, and V. Kreinovich, "Non-traditional neural networks that solve one more intractable problem: propositional satisfiability", Proceedings of the First International Conference on Neural, Parallel, and Scientific Computations, Atlanta, GA, May 28-31, 1995, Vol. 1, pp. $427-430$.

[64] J. Sokolowski and J.-P. Zolesio, Introduction to shape optimization, Springer-Verlag, 1992.

[65] Á. Száz, "The fundamental theorem of calculus in an abstract setting", Tatra Mountains Math. Publ., 1993, Vol. 2, pp. 167-174.

[66] K. Tanaka and Y. Maruyama, "The multiobjective optimization problem of set functions", Journal of Information and Optimization Sciences, 1984, Vol. 5, pp. 293-306.

[67] S. A. Vavasis, Nonlinear optimization: complexity issues, Oxford University Press, N.Y., 1991.

[68] M. I. Zakharevich, "Ergodic properties of Maslov's iterative method", In: Problems of Cybernetics, Moscow, 1987, Vol. 131, pp. 63-76 (in Russian); English translation in: V. Kreinovich and G. Mints (eds.), Problems of reducing the exhaustive search, American Mathematical Society, Providence, RI, 1997, pp. 53-64. 